\title{
Dynamical Analysis on Single Degree-of-Freedom Semiactive Control System by Using Fractional-Order Derivative
}

\author{
Yongjun Shen, ${ }^{1}$ Minghui Fan, ${ }^{1}$ Xianghong Li, ${ }^{2}$ Shaopu Yang, ${ }^{1}$ and Haijun Xing ${ }^{1}$ \\ ${ }^{1}$ Department of Mechanical Engineering, Shijiazhuang Tiedao University, No. 17 Bei Erhuan Dong Road, Shijiazhuang 050043, China \\ ${ }^{2}$ Department of Mathematics and Physics, Shijiazhuang Tiedao University, No. 17 Bei Erhuan Dong Road, Shijiazhuang 050043, China \\ Correspondence should be addressed to Yongjun Shen; shenyongjun@126.com
}

Received 27 January 2015; Revised 22 March 2015; Accepted 23 March 2015

Academic Editor: Roman Lewandowski

Copyright (c) 2015 Yongjun Shen et al. This is an open access article distributed under the Creative Commons Attribution License, which permits unrestricted use, distribution, and reproduction in any medium, provided the original work is properly cited.

\begin{abstract}
The single degree-of-freedom (SDOF) system under the control of three semiactive methods is analytically studied in this paper, where a fractional-order derivative is used in the mathematical model. The three semiactive control methods are on-off control, limited relative displacement (LRD) control, and relative control, respectively. The averaging method is adopted to provide an analytical study on the performance of the three different control methods. Based on the comparison between the analytical solutions with the numerical ones, it could be proved that the analytical solutions are accurate enough. The effects of the fractionalorder parameters on the control performance, especially the relative and absolute displacement transmissibility, are analyzed. The research results indicate that the steady-state amplitudes of the three semiactive systems with fractional-order derivative in the model could be significantly reduced and the control performance can be greatly improved.
\end{abstract}

\section{Introduction}

Machinery equipment will vibrate if it is excited, and more damage can be caused when it is under the condition of resonance. In order to reduce vibration, Frahm invented the original shock absorber based on classical vibration theory. Then Den Hartog propounded the design rule for damping dynamic shock absorber based on the fixed-points theory [1]. Subsequently, many improved shock absorbers were presented based on this one. At that stage, the control method should be named as passive control one. The damping force of passive vibration control system could not be adjusted instantaneously, so that it may be limited effective. With the development of science and technology, active control and semiactive control methods came into being. These two kinds of control methods performed noticeably well and they were adopted in many fields such as vehicle suspension and vibration absorption system [2-5].

Vehicle suspension could be simplified to a single degreeof-freedom (SDOF) semiactive control system under some assumptions. The study on semiactive control methods originated from Karnopp et al. who presented the on-off control method in 1974 [2]. After that, many scholars carried out research directing at semiactive suspension system, and the research efforts were focused on the control methods and/or design of control devices [6-8]. Some other scholars preferred analytical method than numerical one, because the analytical study may present more information about the adjustment or design of the controllable parameters. Shen et al. [9] applied averaging method to present analytical investigations for different control methods. Eslaminasab et al. [10] adopted averaging method to provide an analytical platform for analyzing the performance of relative control method. Shen et al. [11-13] studied the approximately analytical solutions and parameters optimization of four semiactive on-off dynamic vibration absorbers and researched a single degreeof-freedom semiactive oscillator with time delay.

In fact, it is rather hard to accurately describe the constitutive relations of most real materials by the classical integerorder model, especially for some viscoelastic and/or rheological materials. Therefore, some scholars started to research fractional-order derivative models. As far back as 1985, Bagley and Torvik [14-16] introduced fractional-order derivative into fluid mechanics and modelled the constitutive relation of the material viscoelasticity in many vibration mitigations. Rossikhin and Shitikova [17] proposed a method to analyze 
the free damped vibrations of a fractional-order oscillator. Tavazoei et al. [18] and Pinto and Tenreiro Machado [19] studied the fractional-order van der Pol oscillator and found multiple limit cycles existing in the system. Shen et al. [20-23] analytically studied the primary resonance of some nonlinear oscillators with different fractional-order derivatives by the averaging method and illustrated the effects of fractionalorder parameters on dynamical response. Wahi and Chatterjee [24] studied an oscillator with special fractional-order derivative and time-delay by averaging method. Chen and Zhu [25], Wang and He [26], Huang and Jin [27], and Xu et al. [28] also investigated different fractional-order systems and presented some important results by analytical research.

In this paper, we introduce fractional-order derivative into semiactive SDOF systems, where the semiactive methods are on-off control, limited relative displacement (LRD) control, and relative control, respectively. The analytical solutions of the three semiactive systems are obtained by averaging method. Then we study the stability of those steady-state solutions by Lyapunov stability theory. Furthermore, the effects of fractional-order parameters on the steady-state amplitude and control performance are researched. At last the main conclusions are made.

\section{Analytical Solutions of SDOF Semiactive System}

The considered SDOF semiactive system is shown in Figure 1. If the fractional-order viscoelastic device is used, its mathematical model should be

$$
m \ddot{x}+k\left(x-x_{0}\right)+c\left(\dot{x}-\dot{x}_{0}\right)+K D^{p}\left(x-x_{0}\right)=0,
$$

where $x_{0}=X_{0} \cos \omega t$ is the external excitation to the controlled system with $X_{0}$ and $\omega$ as the excitation amplitude and excitation frequency. $m, c$, and $k$ are the system mass, system damping coefficient, and stiffness, respectively. $K D^{p}\left(x-x_{0}\right)$ is the fractional-order derivative of $x-x_{0}$ to $t$ with the fractional coefficient $K(K \geq 0)$ and the fractional-order $p(0 \leq p \leq 1)$. There are several definitions for fractional-order derivative, and they are equivalent under some conditions for a wide class of functions. In Caputo's sense, the definition of $p$ order derivative of $x(t)$ to $t$ is

$$
D^{p}[x(t)]=\frac{1}{\Gamma(1-p)} \int_{0}^{t} \frac{x^{\prime}(u)}{(t-u)^{p}} d u,
$$

where $\Gamma(z)$ is Gamma function that satisfies $\Gamma(z+1)=z \Gamma(z)$.

Defining $z=x-x_{0}$, (1) becomes

$$
m \ddot{z}+k z+c \dot{z}+K D^{p}(z)=m X_{0} \omega^{2} \cos \omega t
$$

Using the following transformation of coordinates

$$
\begin{aligned}
\omega_{0}^{2} & =\frac{k}{m}, \\
\xi & =\frac{c}{2 m},
\end{aligned}
$$

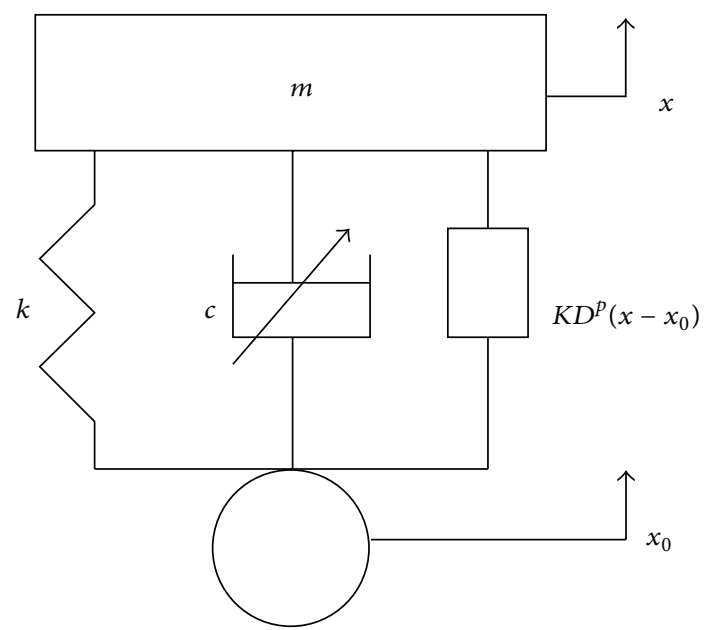

FIGURE 1: The model of SDOF semiactive system by using fractionalorder derivative.

$$
\begin{aligned}
& \lambda=\frac{K}{m}, \\
& f=X_{0} \omega^{2},
\end{aligned}
$$

equation (3) becomes

$$
\ddot{z}+\omega_{0}^{2} z+2 \xi \dot{z}+\lambda D^{p}(z)=f \cos \omega t .
$$

Supposing the solution of (5) is

$$
\begin{aligned}
& z=a \cos \phi, \\
& \dot{z}=-\omega a \sin \phi,
\end{aligned}
$$

where $\phi=\omega t+\theta$, one could get

$$
\begin{aligned}
& \dot{z}=\dot{a} \cos \phi-a(\omega+\dot{\theta}) \sin \phi, \\
& \ddot{z}=-\omega[\dot{a} \sin \phi+a(\omega+\dot{\theta}) \cos \phi] .
\end{aligned}
$$

From (5), (6), and (7), one could obtain

$$
\begin{aligned}
& \dot{a} \cos \phi-a \dot{\theta} \sin \phi=0, \\
& \dot{a} \sin \phi+a \dot{\theta} \cos \phi=\frac{F}{\omega},
\end{aligned}
$$

where

$$
\begin{aligned}
F & =-\left[f \cos \omega t+a\left(\omega^{2}-\omega_{0}^{2}\right) \cos \phi+2 a \xi \omega \sin \phi\right. \\
& \left.-\lambda D^{p}(a \cos \phi)\right] .
\end{aligned}
$$

The derivatives of the generalized amplitude $a$ and phase $\theta$ could be solved as

$$
\begin{aligned}
\dot{a} & =\frac{F}{\omega} \sin \phi, \\
a \dot{\theta} & =\frac{F}{\omega} \cos \phi .
\end{aligned}
$$


Then, one could apply the standard averaging method to (10) in time interval $[0, T]$

$$
\begin{aligned}
\dot{a} & =\lim _{T \rightarrow \infty} \frac{1}{T} \int_{0}^{T} \frac{F}{\omega} \sin \phi d \phi, \\
a \dot{\theta} & =\lim _{T \rightarrow \infty} \frac{1}{T} \int_{0}^{T} \frac{F}{\omega} \cos \phi d \phi .
\end{aligned}
$$

Furthermore, (11) could be written as

$$
\begin{gathered}
\dot{a}=\dot{a}_{1}+\dot{a}_{2}+\dot{a}_{3}, \\
a \dot{\theta}=a \dot{\theta}_{1}+a \dot{\theta}_{2}+a \dot{\theta}_{3},
\end{gathered}
$$

where

$$
\begin{aligned}
\dot{a}_{1} & =\lim _{T \rightarrow \infty} \frac{1}{T \omega} \\
\cdot & \int_{0}^{T}-\left[X_{0} \omega^{2} \cos (\phi-\theta)+a\left(\omega^{2}-\omega_{0}^{2}\right) \cos \phi\right] \\
& \cdot \sin \phi d \phi, \\
\dot{a}_{2} & =\lim _{T \rightarrow \infty} \frac{1}{T \omega} \int_{0}^{T}-2 a \xi \omega \sin ^{2} \phi d \phi, \\
\dot{a}_{3}= & \lim _{T \rightarrow \infty} \frac{1}{T \omega} \int_{0}^{T} \lambda D^{p}(a \cos \phi) \sin \phi d \phi, \\
a \dot{\theta}_{1} & =\lim _{T \rightarrow \infty} \frac{1}{T \omega} \\
& \cdot \int_{0}^{T}-\left[X \omega_{0}^{2} \cos (\phi-\theta)+a\left(\omega^{2}-\omega_{0}^{2}\right) \cos \phi\right] \\
& \cdot \cos \phi d \phi, \\
a \dot{\theta}_{3} & =\lim _{T \rightarrow \infty} \frac{1}{T \omega} \int_{0}^{T} \lambda D^{p}(a \cos \phi) \cos \phi d \phi . \\
\dot{\theta}_{2} & -a \xi \omega \sin 2 \phi d \phi,
\end{aligned}
$$

According to the averaging method, one could select the time terminal $T$ as $T=2 \pi$ if the integrand is periodic function or $T=\infty$ if the integrand is aperiodic one. Accordingly, one could obtain

$$
\begin{aligned}
\dot{a}_{1} & =-\frac{X_{0} \omega \sin \theta}{2} \\
a \dot{\theta}_{1} & =\frac{-a\left(\omega^{2}+\omega_{0}^{2}\right)-X_{0} \omega^{2} \cos \theta}{2 \omega} .
\end{aligned}
$$

In order to obtain the definite integral in (13c) and (14c), two important formulae, which have been deduced in $[20,21]$, are introduced

$$
\begin{aligned}
B_{1} & =\lim _{T \rightarrow \infty} \int_{0}^{T} \frac{\sin (\omega t)}{t^{p}} d t \\
& =\omega^{p-1} \Gamma(1-p) \cos \left(\frac{p \pi}{2}\right),
\end{aligned}
$$

$$
\begin{aligned}
B_{2} & =\lim _{T \rightarrow \infty} \int_{0}^{T} \frac{\cos (\omega t)}{t^{p}} d t \\
& =\omega^{p-1} \Gamma(1-p) \sin \left(\frac{p \pi}{2}\right) .
\end{aligned}
$$

Based on Caputo's definition, (13c) becomes

$$
\begin{aligned}
\dot{a}_{3} & =\lim _{T \rightarrow \infty} \frac{\lambda}{T \omega} \int_{0}^{T} D^{p}[a \cos (\omega t+\theta)] \sin (\omega t+\theta) d t \\
& =\frac{-\lambda a}{\Gamma(1-p)} \lim _{T \rightarrow \infty} \frac{1}{T} \\
& \cdot \int_{0}^{T}\left\{\left[\int_{0}^{t} \frac{\sin (\omega u+\theta)}{(t-u)^{p}} d u\right] \sin (\omega t+\theta)\right\} d t .
\end{aligned}
$$

Introducing $s=t-u$ and $d s=-d u$, (17) becomes

$$
\begin{aligned}
\dot{a}_{3} & =\frac{-\lambda a}{\Gamma(1-p)} \lim _{T \rightarrow \infty} \frac{1}{T} \int_{0}^{T}\left\{\left[\int_{0}^{t} \frac{\sin (\omega t+\theta-\omega s)}{s^{p}} d s\right]\right. \\
& \cdot \sin (\omega t+\theta)\} d t=\frac{-\lambda a}{\Gamma(1-p)} \lim _{T \rightarrow \infty} \frac{1}{T} \\
& \cdot \int_{0}^{T}\left\{\left[\int_{0}^{t} \frac{\cos (\omega s)}{s^{p}} d s\right] \sin (\omega t+\theta) \sin (\omega t+\theta)\right\} d t \\
& +\frac{\lambda a}{\Gamma(1-p)} \lim _{T \rightarrow \infty} \frac{1}{T} \int_{0}^{T}\left\{\left[\int_{0}^{t} \frac{\sin (\omega s)}{s^{p}} d s\right]\right. \\
& \cdot \cos (\omega t+\theta) \sin (\omega t+\theta)\} d t .
\end{aligned}
$$

In fact, the second part in (18) would vanish according to (16a) and (16b). Defining the first part in (18) as $A_{1}$ and integrating it by parts, one could obtain

$$
\begin{aligned}
A_{1} & \left.\frac{-\lambda a}{4 \omega \Gamma(1-p)} \lim _{T \rightarrow \infty}\left\{\frac{2 \omega t-\sin (2 \omega t+2 \theta)}{T}\left[\int_{0}^{t} \frac{\cos (\omega s)}{s^{p}} d s\right]\right\}\right|_{0} ^{T} \\
& -\frac{-\lambda a}{4 \omega \Gamma(1-p)} \lim _{T \rightarrow \infty} \frac{1}{T} \int_{0}^{T}\left[\frac{[2 \omega t-\sin (2 \omega t+2 \theta)] \cos (\omega t)}{t^{p}}\right] d t .
\end{aligned}
$$

Based on (16a) and (16b), one could find that the second part in (19) would vanish and the first part in (19) will be

$$
A_{1}=-\frac{-\lambda a \omega^{p-1}}{2} \sin \left(\frac{p \pi}{2}\right) \text {. }
$$

That means

$$
\dot{a}_{3}=\frac{-\lambda a \omega^{p-1}}{2} \sin \left(\frac{p \pi}{2}\right) .
$$

After the similar procedure, (14c) will be

$$
a \dot{\theta}_{3}=\frac{\lambda a \omega^{p-1}}{2} \cos \left(\frac{p \pi}{2}\right) .
$$


Equations (13b) and (14b) can be rewritten as

$$
\begin{array}{r}
\dot{a}_{2}=\frac{1}{2 \pi} \int_{0}^{2 \pi}-2 a \xi \sin ^{2} \phi d \phi, \\
a \dot{\theta}_{2}=\frac{1}{2 \pi} \int_{0}^{2 \pi}-a \xi \sin 2 \phi d \phi .
\end{array}
$$

The solutions of (23a) and (23b) depend on the specific semiactive control methods.

Accordingly, (12) becomes

$$
\begin{aligned}
\dot{a}= & -\frac{X_{0} \omega \sin \theta}{2}-\frac{\lambda a \omega^{p-1}}{2} \sin \left(\frac{p \pi}{2}\right) \\
& +\frac{a}{2 \pi} \int_{0}^{2 \pi}-2 \xi \sin ^{2} \phi d \phi, \\
a \dot{\theta}= & \frac{-a \omega^{2}+a \omega_{0}^{2}-X_{0} \omega^{2} \cos \theta}{2 \omega}+\frac{\lambda a \omega^{p-1}}{2} \cos \left(\frac{p \pi}{2}\right) \\
& +\frac{a}{2 \pi} \int_{0}^{2 \pi}-\xi \sin 2 \phi d \phi,
\end{aligned}
$$

which could be rewritten as

$$
\begin{aligned}
\dot{a}= & -\frac{X_{0} \omega \sin \theta}{2}-\frac{a}{2 m} C^{\prime}, \\
a \dot{\theta}= & \frac{-X_{0} \omega \cos \theta}{2}-\frac{\omega a}{2}+\frac{a}{2 \pi} \int_{0}^{2 \pi}-\frac{c}{2 m} \sin 2 \phi d \phi \\
& +\frac{a}{2 m \omega} K^{\prime},
\end{aligned}
$$

where

$$
\begin{aligned}
& C^{\prime}=K \omega^{p-1} \sin \left(\frac{p \pi}{2}\right)+\int_{0}^{2 \pi} \frac{-c \sin ^{2} \phi d \phi}{\pi}, \\
& K^{\prime}=K \omega^{p} \cos \left(\frac{p \pi}{2}\right)+k .
\end{aligned}
$$

$C^{\prime}$ and $K^{\prime}$ are defined as the equivalent damping coefficient and equivalent stiffness coefficient in semiactive systems, respectively. In the SDOF semiactive system, the fractionalorder derivative $K D^{p}\left(x-x_{0}\right)$ serves as damping and stiffness at the same time. When $p \rightarrow 0, K D^{p}\left(x-x_{0}\right)$ will be changed into spring force, whereas it will be almost the same as damping force if $p \rightarrow 1$. When $K \rightarrow 0, K D^{p}\left(x-x_{0}\right)$ will have little effect on control performance and the semiactive system will degrade into the traditional semiactive one.

\section{Cases for Three Kinds of \\ Semiactive Control Systems}

3.1. Analytical Investigation for on-off Control System. The strategy of semiactive on-off control is

$$
c= \begin{cases}c_{\max } & \dot{x}\left(\dot{x}-\dot{x}_{0}\right) \geq 0, \\ c_{\min } & \text { else. }\end{cases}
$$

Originally, $c_{\min }$ is selected as $c_{\min }=0$ in [2], which may be inaccurate in real engineering. Here we select it as $c_{\min }=$ $0.01 c_{\max }$.

After transformation of coordinates, (27) becomes

$$
\xi= \begin{cases}\xi_{\max } & \dot{z}\left(\dot{z}+\dot{x}_{0}\right) \geq 0, \\ \xi_{\min } & \text { else. }\end{cases}
$$

Expanding the critical condition

$$
\left(\dot{z}+\dot{x}_{0}\right) \dot{z} \geq 0
$$

one could get

$$
\begin{gathered}
-\omega a \sin \phi\left[-\omega a \sin \phi-X_{0} \omega \sin (\omega t)\right] \\
=a R \sin \phi \sin (\phi-\beta) \geq 0,
\end{gathered}
$$

where

$$
\begin{aligned}
& \beta=\arctan \frac{X_{0} \sin \theta}{a+X_{0} \cos \theta}, \\
& R=\omega^{2} \sqrt{\left(a+X_{0} \cos \theta\right)^{2}+X_{0}^{2} \sin ^{2} \theta} .
\end{aligned}
$$

Equation (28) can be rewritten as

$$
\xi= \begin{cases}\xi_{\min } & 0<\phi<\beta, \\ \xi_{\max } & \beta<\phi<\pi, \\ \xi_{\min } & \pi<\phi<\pi+\beta, \\ \xi_{\max } & \pi+\beta<\phi<2 \pi .\end{cases}
$$

Using the averaging method, one could obtain the simplified forms of (13b) and (14b) as

$$
\begin{aligned}
\dot{a}_{2} & =\frac{-2 a A-a B}{2 \pi}, \\
a \dot{\theta}_{2} & =\frac{a C-a D}{2 \pi},
\end{aligned}
$$

where

$$
\begin{aligned}
& A=\pi \xi_{\text {max }}+\beta\left(-\xi_{\max }+\xi_{\text {min }}\right), \\
& B=\left(\xi_{\text {max }}-\xi_{\text {min }}\right) \sin 2 \beta, \\
& C=\xi_{\text {max }}-\xi_{\text {min }}, \\
& D=\left(\xi_{\text {max }}-\xi_{\text {min }}\right) \cos 2 \beta .
\end{aligned}
$$

Combining (12), (15a), (15b), (21), (22), and (33), one could obtain

$$
\begin{aligned}
\dot{a}= & -\frac{X_{0} \omega \sin \theta}{2}-\frac{\lambda a \omega^{p-1}}{2} \sin \left(\frac{p \pi}{2}\right)+\frac{-2 a A-a B}{2 \pi}, \\
a \dot{\theta}= & \frac{-a \omega^{2}+a \omega_{0}^{2}-X_{0} \omega^{2} \cos \theta}{2 \omega}+\frac{\lambda a \omega^{p-1}}{2} \cos \left(\frac{p \pi}{2}\right) \\
& +\frac{a C-a D}{2 \pi} .
\end{aligned}
$$


Obviously, the equivalent damping coefficient in this case could be written as

$$
\begin{aligned}
C^{\prime}= & K \omega^{p-1} \sin \left(\frac{p \pi}{2}\right)+c_{\max }+\frac{\beta}{\pi}\left(-c_{\max }+c_{\min }\right) \\
& +\frac{\sin 2 \beta}{2 \pi}\left(c_{\max }-c_{\min }\right) .
\end{aligned}
$$

From (36), it could be found that the equivalent damping coefficient $C^{\prime}$ mainly depends on the fractional-order parameters $K$ and $p$, associated with the critical angle $\beta$.

Now we study the steady-state solution, which is more important and meaningful in vibration engineering. Letting $\dot{a}=0$ and $a \dot{\theta}=0$, one could obtain the amplitude and phase of steady-state response

$$
\begin{aligned}
& \bar{a}=\frac{\pi X_{0} \omega^{2}}{\sqrt{N^{2}+L^{2}}}, \\
& \bar{\theta}=\arctan \frac{N}{L},
\end{aligned}
$$

where

$$
\begin{aligned}
& N=-2 \omega A-\omega B-\pi \omega^{p} \lambda \sin \frac{p \pi}{2} \\
& L=\omega C-\omega D+\pi \omega^{p} \lambda \cos \frac{p \pi}{2}+\pi \omega_{0}^{2}-\pi \omega^{2} .
\end{aligned}
$$

The transmissibility for relative displacement is

$$
\mu_{1}=\left|\frac{\bar{a}}{X_{0}}\right|=\frac{\pi \omega^{2}}{\sqrt{N^{2}+L^{2}}} .
$$

The absolute displacement of on-off control system is

$$
\begin{aligned}
x & =x_{0}+z=X_{0} \cos \omega t+a \cos (\omega t+\theta) \\
& =\bar{X}_{1} \cos (\omega t-\tau),
\end{aligned}
$$

where

$$
\begin{aligned}
& \bar{X}_{1}=\sqrt{\frac{X_{0}^{2} \pi^{2} \omega^{4} N^{2}}{\left(N^{2}+L^{2}\right)^{2}}+\left(X_{0}+\frac{X_{0} \pi \omega^{2}|L|}{N^{2}+L^{2}}\right)^{2}}, \\
& \tau=-\arctan \frac{\pi \omega^{2} N}{L\left(\pi \omega^{2}+\left(L^{2}+N^{2}\right) /|L|\right)} .
\end{aligned}
$$

The displacement transmissibility is

$$
\varsigma_{1}=\left|\frac{x}{X_{0}}\right|=\frac{\bar{X}_{1}}{X_{0}} .
$$

Next, we study the stability of steady-state solution. Letting $a=\bar{a}+\Delta a$ and $\theta=\bar{\theta}+\Delta \theta$ and then substituting them into (35), one could get

$$
\begin{aligned}
& \frac{d \Delta a}{d t}=\frac{1}{2 \pi}\left[N \cdot \Delta a-X_{0} \pi \omega \cos \bar{\theta} \Delta \theta\right], \\
& \frac{d \Delta \theta}{d t}=\frac{1}{2 \pi}\left[\frac{\pi X_{0} \omega \sin \bar{\theta}}{\bar{a}} \cdot \Delta \theta+\frac{\pi X_{0} \omega \cos \bar{\theta}}{\bar{a}^{2}} \cdot \Delta a\right] .
\end{aligned}
$$

Based on (37), one could eliminate $\bar{\theta}$ and obtain the characteristic determinant

$$
\operatorname{det}\left[\begin{array}{cc}
\frac{N}{\omega}-\alpha & \frac{-\bar{a} L}{\omega} \\
\frac{L}{\bar{a} \omega} & \frac{N}{\omega}-\alpha
\end{array}\right]=0 .
$$

Expanding the determinant, one could obtain the characteristic equation

$$
\left(\frac{N}{\omega}-\alpha\right)^{2}+\frac{L^{2}}{\omega^{2}}=0 .
$$

Solving (45), one could obtain characteristic values

$$
\alpha_{1,2}=\frac{N}{\omega} \pm \frac{L}{\omega} i
$$

Obviously, $N$ is less than 0 . Accordingly, all real parts of characteristic roots are negative, which means the steady-state solution of this kind of semiactive system is unconditionally stable.

3.2. Analytical Investigation for LRD Control System. The strategy of LRD control is

$$
c= \begin{cases}c_{\max } & \left|x-x_{0}\right| \geq \delta, \\ c_{\min } & \left|x-x_{0}\right|<\delta,\end{cases}
$$

where $c_{\min }=0.01 c_{\max }$ and $\delta$ is the space between the mass and excitation point. Here we select $\delta=0.5 X_{0}$.

After transformation of coordinates, (47) becomes

$$
c= \begin{cases}c_{\max } & |z| \geq \delta, \\ c_{\min } & |z|<\delta,\end{cases}
$$

and the control strategy can be rewritten as

$$
\xi= \begin{cases}\xi_{\max } & 0<\phi<\varepsilon, \\ \xi_{\min } & \varepsilon<\phi<\pi-\varepsilon, \\ \xi_{\max } & \pi-\varepsilon<\phi<\pi+\varepsilon, \\ \xi_{\min } & \pi+\varepsilon<\phi<2 \pi-\varepsilon, \\ \xi_{\max } & 2 \pi-\varepsilon<\phi<2 \pi,\end{cases}
$$

where $\varepsilon=\arccos \left(0.5 X_{0} / a\right)$.

Using the averaging method, one could obtain the simplified forms of (13b) and (14b)

$$
\begin{aligned}
\dot{a}_{2} & =\frac{-2 a A_{0}+2 a B_{0}}{2 \pi \omega}, \\
a \dot{\theta}_{2} & =0,
\end{aligned}
$$

where $A_{0}=\omega\left(2 \varepsilon \xi_{\max }+\pi \xi_{\min }-2 \varepsilon \xi_{\min }\right), B_{0}=\omega\left(\xi_{\max }-\right.$ $\left.\xi_{\min }\right) \sin 2 \varepsilon$. 
Combining (12), (15a), (15b), (21), (22), and (50), one could obtain

$$
\begin{aligned}
\dot{a}= & \frac{-2 a A_{0}+2 a B_{0}-X_{0} \omega^{2} \pi \sin \theta}{2 \pi \omega} \\
& -\frac{\lambda a \omega^{p-1}}{2} \sin \left(\frac{p \pi}{2}\right), \\
a \dot{\theta}= & \frac{-a \pi\left(\omega^{2}-\omega_{0}^{2}\right)-X_{0} \omega^{2} \pi \cos \theta}{2 \pi \omega} \\
& +\frac{\lambda a \omega^{p-1}}{2} \cos \left(\frac{p \pi}{2}\right) .
\end{aligned}
$$

Obviously, one could easily obtain the equivalent damping coefficient

$$
\begin{aligned}
& C^{\prime} \\
& =K \omega^{p-1} \sin \left(\frac{p \pi}{2}\right) \\
& \quad+\frac{\left(c_{\max }-c_{\min }\right) \sin 2 \varepsilon-\left(2 \varepsilon c_{\max }+c_{\min } \pi-2 \varepsilon c_{\min }\right)}{\pi} .
\end{aligned}
$$

From (52), it could be found that the equivalent damping coefficient $C^{\prime}$ mainly depends on the fractional-order parameters $K$ and $p$, associated with the critical angle $\varepsilon$.

Now we study the steady-state solution, which is more important and meaningful in vibration engineering. Letting $\dot{a}=0$ and $a \dot{\theta}=0$, one could obtain

$$
\begin{aligned}
& \bar{a}=\frac{\pi X_{0} \omega^{2}}{\sqrt{\left[-2 A_{0}+2 B_{0}-\lambda \pi \omega^{p} \sin (p \pi / 2)\right]^{2}+\left[-\pi\left(\omega^{2}-\omega_{0}^{2}\right)+\lambda \pi \omega^{p} \cos (p \pi / 2)\right]^{2}}}, \\
& \bar{\theta}=\arctan \frac{-2 A_{0}+2 B_{0}-\lambda \pi \omega^{p} \sin (p \pi / 2)}{-\pi\left(\omega^{2}-\omega_{0}^{2}\right)+\lambda \pi \omega^{p} \cos (p \pi / 2)}
\end{aligned}
$$

where $\bar{a}$ and $\bar{\theta}$ are the amplitude and phase of steady-state response, respectively.

The transmissibility for relative displacement is

$$
\mu_{2}=\left|\frac{\bar{a}}{X_{0}}\right|=\frac{\pi \omega^{2}}{\sqrt{\left[-2 A_{0}+2 B_{0}-\lambda \pi \omega^{p} \sin (p \pi / 2)\right]^{2}+\left[-\pi\left(\omega^{2}-\omega_{0}^{2}\right)+\lambda \pi \omega^{p} \cos (p \pi / 2)\right]^{2}}} .
$$

The absolute displacement of LRD control system is

$$
\begin{aligned}
x & =x_{0}+z=X_{0} \cos \omega t+a \cos (\omega t+\theta) \\
& =\bar{X}_{2} \cos (\omega t-\chi),
\end{aligned}
$$

where

$$
\begin{aligned}
\bar{X}_{2}^{2} & \\
= & \frac{X_{0}^{2} \pi^{2} \omega^{4} W^{2}}{\left[\left(2 A_{0}-2 B_{0}\right)^{2}+\pi^{2}\left(\omega^{4}-2 \omega^{2} \omega_{0}^{2}+\omega_{0}^{4}+\omega^{2 p} \lambda^{2}\right)-2 \pi^{2} \omega^{p}\left(\omega^{2}-\omega_{0}^{2}\right) \lambda \cos (p \pi / 2)+4\left(A_{0}-B_{0}\right) \pi \omega^{p} \lambda \sin (p \pi / 2)\right]^{2}} \\
& +\left(X_{0}+\frac{X_{0} \pi \omega^{2} G}{G^{2}+W^{2}}\right)^{2} \\
\chi= & \arctan \frac{\pi^{2} \omega^{2} W}{G \pi^{2} \omega^{2}+\pi G^{2}+\pi W^{2}},
\end{aligned}
$$


where

$$
\begin{aligned}
& G=\pi\left(-\omega^{2}+\omega_{0}^{2}+\omega^{p} \lambda \cos \frac{p \pi}{2}\right), \\
& W=2 A_{0}-2 B_{0}+\pi \omega^{p} \lambda \sin \frac{p \pi}{2} .
\end{aligned}
$$

The transmissibility for the absolute displacement of LRD control system is

$$
\varsigma_{2}=\left|\frac{x}{X_{0}}\right|=\frac{\bar{X}_{2}}{X_{0}}
$$

Next, we study the stability of the steady-state solutions. Letting $a=\bar{a}+\Delta a$ and $\theta=\bar{\theta}+\Delta \theta$ and substituting them into (51), one could get

$$
\begin{aligned}
& \frac{d \Delta a}{d t}=\frac{1}{2 \pi \omega}\left[-W \cdot \Delta a-X_{0} \pi \omega^{2} \cos \bar{\theta} \Delta \theta\right], \\
& \frac{d \Delta \theta}{d t} \\
& \quad=\frac{1}{2 \pi \omega}\left[\frac{\pi X_{0} \omega^{2} \sin \bar{\theta}}{\bar{a}} \cdot \Delta \theta+\frac{\pi X_{0} \omega^{2} \cos \bar{\theta}}{\bar{a}^{2}} \cdot \Delta a\right] .
\end{aligned}
$$

Based on (53), one could eliminate $\bar{\theta}$ and obtain the characteristic determinant as

$$
\operatorname{det}\left[\begin{array}{cc}
-W-\alpha & -\bar{a} G \\
\frac{G}{\bar{a}} & -W-\alpha
\end{array}\right]=0 .
$$

Expanding the determinant one could obtain the characteristic equation as

$$
(-W-\alpha)^{2}+G^{2}=0 .
$$

Solving (61), one could obtain the characteristic values as

$$
\alpha_{1,2}=-W \pm G i
$$

Obviously, all real parts of the characteristic roots are negative, which means the steady-state solution of this kind of semiactive system is also unconditionally stable.

3.3. Analytical Investigation for Relative Control System. The strategy for semiactive relative control is shown as

$$
c= \begin{cases}c_{\max } & \left(x-x_{0}\right)\left(\dot{x}-\dot{x}_{0}\right) \leq 0, \\ c_{\min } & \text { else. }\end{cases}
$$

After transformation of coordinates, (63) will become

$$
\xi= \begin{cases}\xi_{\max } & z \dot{z} \leq 0, \\ \xi_{\min } & \text { else }\end{cases}
$$

and the control strategy can be rewritten as

$$
\xi= \begin{cases}\xi_{\max } & 0<\phi<\frac{\pi}{2}, \\ \xi_{\min } & \frac{\pi}{2}<\phi<\pi, \\ \xi_{\max } & \pi<\phi<\frac{3 \pi}{2}, \\ \xi_{\min } & \frac{3 \pi}{2}<\phi<2 \pi .\end{cases}
$$

Using the averaging method, one could obtain the simplified forms of (13b) and (14b) for this semiactive control system

$$
\begin{array}{r}
\dot{a}_{2}=\frac{-\pi a\left(\xi_{\max }+\xi_{\min }\right)}{2 \pi}, \\
a \dot{\theta}_{2}=\frac{2 a\left(-\xi_{\max }+\xi_{\min }\right)}{2 \pi} .
\end{array}
$$

Combining (12), (15a), (15b), (21), (22), and (66), one could obtain

$\dot{a}$

$$
\begin{aligned}
= & \frac{-\pi \omega a\left(\xi_{\max }+\xi_{\min }\right)-X_{0} \pi \omega^{2} \sin \theta}{2 \pi \omega} \\
& -\frac{\lambda a \omega^{p-1}}{2} \sin \left(\frac{p \pi}{2}\right),
\end{aligned}
$$

$a \dot{\theta}$

$$
\begin{aligned}
= & \frac{a\left[\pi\left(\omega_{0}^{2}-\omega^{2}\right)+2 \omega\left(-\xi_{\max }+\xi_{\min }\right)\right]-X_{0} \pi \omega^{2} \cos \theta}{2 \pi \omega} \\
& +\frac{\lambda a \omega^{p-1}}{2} \cos \left(\frac{p \pi}{2}\right) .
\end{aligned}
$$

Obviously, we could easily obtain the equivalent damping coefficient

$$
C^{\prime}=K \omega^{p-1} \sin \left(\frac{p \pi}{2}\right)+\frac{c_{\max }+c_{\min }}{2} .
$$

From (68), we can find that the equivalent damping coefficient $C^{\prime}$ mainly depends on the fractional-order parameters $K$ and $p$, associated with the original damping coefficient.

Letting $\dot{a}=0$ and $a \dot{\theta}=0$, one could obtain the amplitude and phase of the steady-state response

$$
\begin{aligned}
& \bar{a}=\frac{\pi X_{0} \omega^{2}}{\sqrt{U^{2}+V^{2}}}, \\
& \bar{\theta}=\arctan \frac{U}{V},
\end{aligned}
$$

where

$$
\begin{aligned}
U= & -\lambda \pi \omega^{p} \sin \left(\frac{p \pi}{2}\right)-\pi \omega\left(\xi_{\max }+\xi_{\min }\right), \\
V= & \pi\left(\omega_{0}^{2}-\omega^{2}\right)+2 \omega\left(-\xi_{\max }+\xi_{\min }\right) \\
& +\lambda \pi \omega^{p} \cos \left(\frac{p \pi}{2}\right) .
\end{aligned}
$$



is

The transmissibility for relative displacement in this case

$$
\mu_{3}=\left|\frac{\bar{a}}{X_{0}}\right|=\frac{\pi \omega^{2}}{\sqrt{U^{2}+V^{2}}}
$$

The absolute displacement of relative control system is

$$
\begin{aligned}
x & =x_{0}+z=X_{0} \cos \omega t+a \cos (\omega t+\theta) \\
& =\bar{X}_{3} \cos (\omega t-\gamma),
\end{aligned}
$$

where

$$
\begin{aligned}
& \bar{X}_{3}=\sqrt{\left(X_{0}+\frac{X_{0} \pi \omega^{2} \cos \psi}{\sqrt{Q}}\right)^{2}+\frac{X_{0}^{2} \pi^{2} \omega^{4} \sin ^{2} \psi}{Q}}, \\
& \gamma=\arctan \frac{X_{0} \pi \omega^{2} \sin \psi}{\left(X_{0}+\cos \psi\right) \sqrt{Q}},
\end{aligned}
$$

where

$$
\begin{aligned}
\psi & =\frac{U}{V}, \\
Q & =V^{2}+U^{2} .
\end{aligned}
$$

The transmissibility for the absolute displacement is

$$
\varsigma_{3}=\left|\frac{x}{X_{0}}\right|=\frac{\bar{X}_{3}}{X_{0}} .
$$

Next, we study the stability of steady-state solutions. Letting $a=\bar{a}+\Delta a, \theta=\bar{\theta}+\Delta \theta$ and substituting them into (67), one could get

$$
\begin{aligned}
& \frac{d \Delta a}{d t} \\
& =\frac{1}{2 \pi \omega}\left[\left[-\pi \omega\left(\xi_{\max }+\xi_{\min }\right)-\lambda \pi \omega^{p} \sin \left(\frac{p \pi}{2}\right)\right]\right. \\
& \left.\cdot \Delta a-X_{0} \pi \omega^{2} \cos \bar{\theta} \Delta \theta\right], \\
& \frac{d \Delta \theta}{d t}=\frac{1}{2 \pi \omega}\left[\frac{\pi X_{0} \omega^{2} \sin \bar{\theta}}{\bar{a}} \cdot \Delta \theta+\frac{\pi X_{0} \omega^{2} \cos \bar{\theta}}{\bar{a}^{2}}\right. \\
& \cdot \Delta a] .
\end{aligned}
$$

Based on (69), one could eliminate $\bar{\theta}$ and obtain the characteristic determinant:

$$
\operatorname{det}\left[\begin{array}{cc}
U-\alpha & -\bar{a} V \\
\frac{U}{\bar{a}} & V-\alpha
\end{array}\right]=0 .
$$

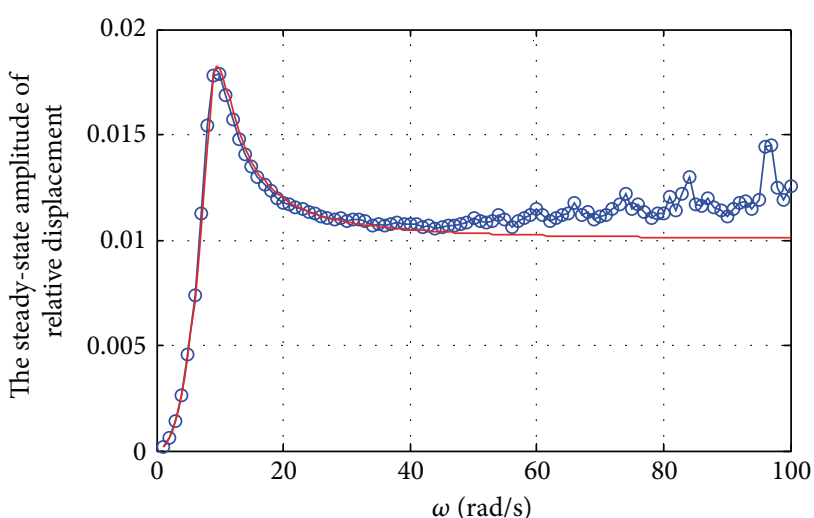

$$
\begin{aligned}
& - \text { Numerical solution } \\
& - \text { Analytical solution }
\end{aligned}
$$

FIgURE 2: Comparison of analytical solution with numerical one under on-off control.

Expanding the determinant, one could obtain the characteristic equation:

$$
(U-\alpha)^{2}+V^{2}=0
$$

Solving (78), one could obtain the characteristic values:

$$
\alpha_{1,2}=U \pm V i
$$

Obviously, $U<0$. That is to say, all real parts of the characteristic values are negative, which means the steady-state solution of this kind of semiactive system is stable unconditionally.

\section{Numerical Simulation and Analysis}

4.1. Comparison between the Analytical and Numerical Solution. In order to verify the precision of the analytical solutions, we also present the numerical results. The numerical scheme [29-34] is

$$
D^{p}\left[x\left(t_{l}\right)\right] \approx h^{-p} \sum_{j=0}^{l} C_{j}^{p} x\left(t_{l-j}\right),
$$

where $t_{l}=l h$ is the time sample points, $h$ is the sample step, and $C_{j}^{p}$ is the fractional binomial coefficient with the iterative relationship as

$$
\begin{aligned}
& C_{0}^{p}=1, \\
& C_{j}^{p}=\left(1-\frac{1+p}{j}\right) C_{j-1}^{p} .
\end{aligned}
$$

The selected basic system parameters are $m=240, k=$ $16000, c=1000, X_{0}=0.01, p=0.5, K=1000$, and $\delta=0.5 X_{0}$. We take 400 seconds as numerical time in the process of 


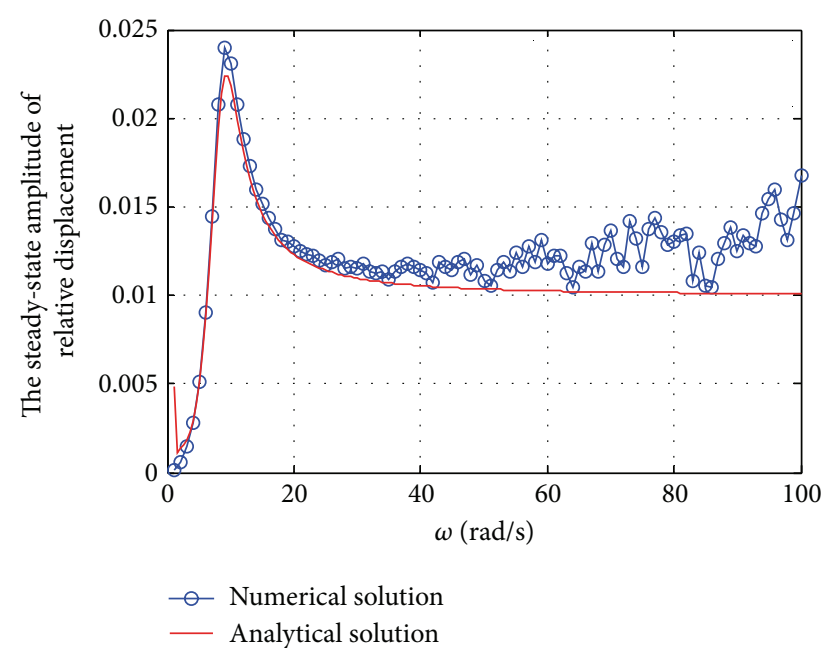

Figure 3: Comparison of analytical solution with numerical one under LRD control.

the simulation. Considering the maximum value of the numerical results in the last 100 seconds as the steady-state amplitude, one could get the amplitude-frequency curves for the numerical results, which are denoted by the lines with circles shown in Figures 2-4. Based on (37), (53), and (69), one could obtain the amplitude-frequency curves by the analytical solutions denoted by the solid lines in Figures 2-4. From the observation of the three figures, one could conclude that the analytical solutions agree very well with the numerical results and could present satisfactory precision. However, there are distinct errors between the approximate analytical and numerical solution in some high-frequency range, especially the amplitude. These phenomena, called chatter in semiactive control system, had also been found by Ahmadian [35] numerically and experimentally.

4.2. Effects of $K$ on the Steady-State Amplitudes. When the fractional coefficient $K$ is changed, the different amplitudefrequency curves are shown in Figures 5-7. From the observation of Figures 5-7, we could conclude that the larger the fractional coefficient $K$ is, the smaller the steady-state amplitudes of the relative and absolute displacement of system mass are. At the same time, the resonant frequency also becomes larger. This phenomenon indicates that both the equivalent damping coefficient and equivalent stiffness coefficient become larger in this procedure. That is to say, the fractional-order derivative $K D^{p}\left(x-x_{0}\right)$ serves as damping and spring simultaneously.

4.3. Effects of $p$ on the Steady-State Amplitudes. When the fractional coefficient $p$ is changed, the results are shown in Figures 8-10. From the observation of Figures 8-10, we could conclude that the larger the fractional coefficient $p$ is, the smaller the steady-state amplitudes of the relative and absolute displacement of system mass are. However, it seems

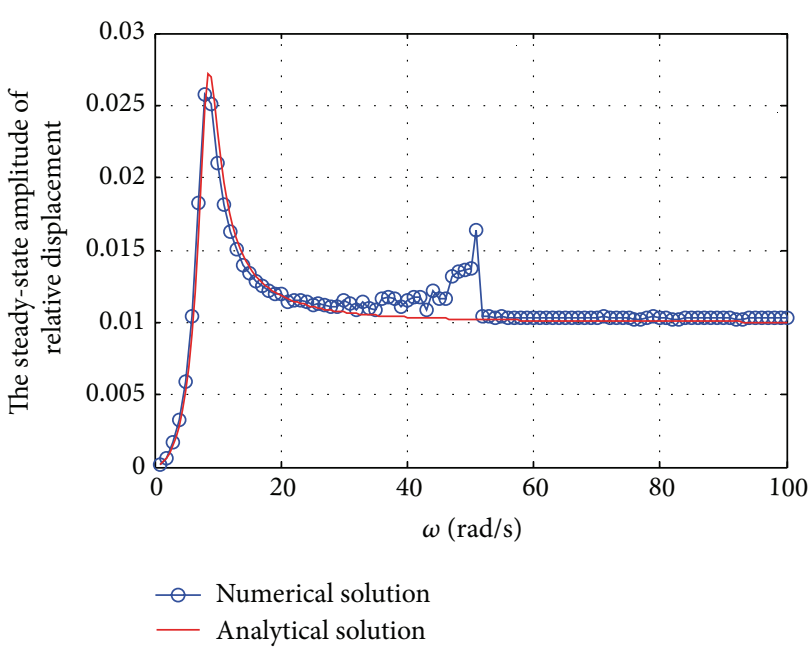

FIGURE 4: Comparison of analytical solution with numerical one under relative control.

that there is little effect of $p$ on the resonant frequency. In fact, by differentiating $K^{\prime}$ and $C^{\prime}$ to $p$, one can get

$$
\begin{aligned}
& \frac{d C^{\prime}}{d p}=k \omega^{p-1} \sqrt{\left(p \omega^{-1}\right)^{2}+\left(\frac{\pi}{2}\right)^{2}} \sin \left(\frac{p \pi}{2}+\vartheta_{1}\right), \\
& \frac{d K^{\prime}}{d p}=-k \omega^{p} \sqrt{\left(p \omega^{-1}\right)^{2}+\left(\frac{\pi}{2}\right)^{2}} \sin \left(\frac{p \pi}{2}-\vartheta_{2}\right),
\end{aligned}
$$

where

$$
\begin{aligned}
& \vartheta_{1}=\arctan \frac{\pi \omega}{2 p}, \\
& \vartheta_{2}=\arctan \frac{2 p}{\pi \omega} .
\end{aligned}
$$

Because $0<p<1$, we can conclude that the derivative of $C^{\prime}$ with respect to $p$ will be larger than $0 . C^{\prime}$ will be monotonically larger if $p$ becomes larger. Accordingly the steadystate amplitude will be smaller in the process. But the value of $\dot{K}^{\prime}$ is not monotonical in this procedure. $K^{\prime}$ could increase or reduce with different $p$, which is affected by the system parameters.

\section{Conclusion}

In this paper, three SDOF semiactive systems are researched by using averaging method. Aiming at these systems, we present the concepts of equivalent damping coefficient and equivalent stiffness coefficient in order to account for the effect of the fractional-order derivative on the control performance.

Based on the above study, one could find that fractionalorder derivative plays an important role in SDOF semiactive system. Therefore, the results in this paper supply valuable reference for engineering practice. In addition, analytical method in this paper could be adopted in two or multidegrees-of-freedom system, and it also offers a new idea to the research field on vehicle suspension theory. 


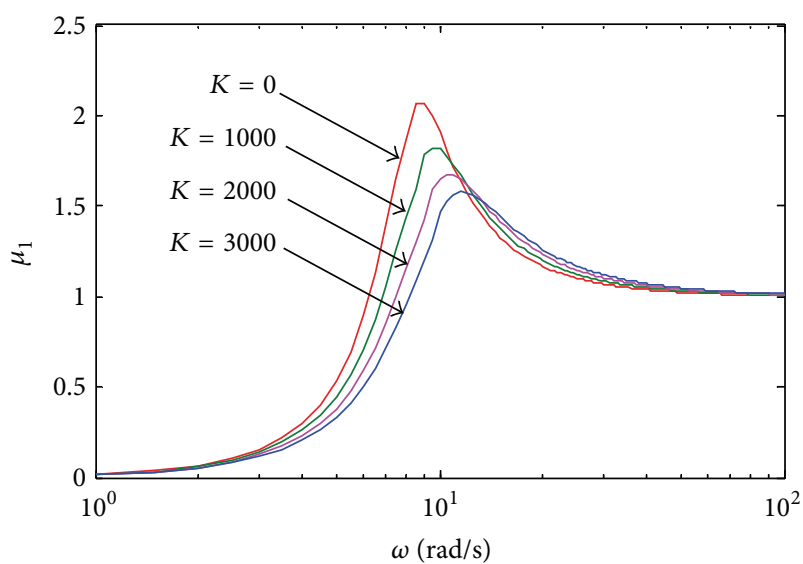

(a) Effect of $K$ on relative displacement transmissibility

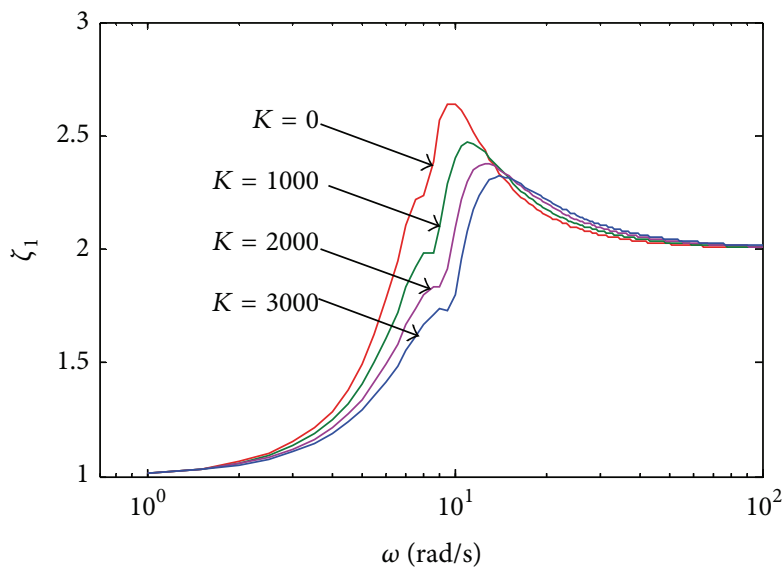

(b) Effect of $K$ on absolute displacement transmissibility

Figure 5: Effect of $K$ on the steady-state amplitudes under on-off control.

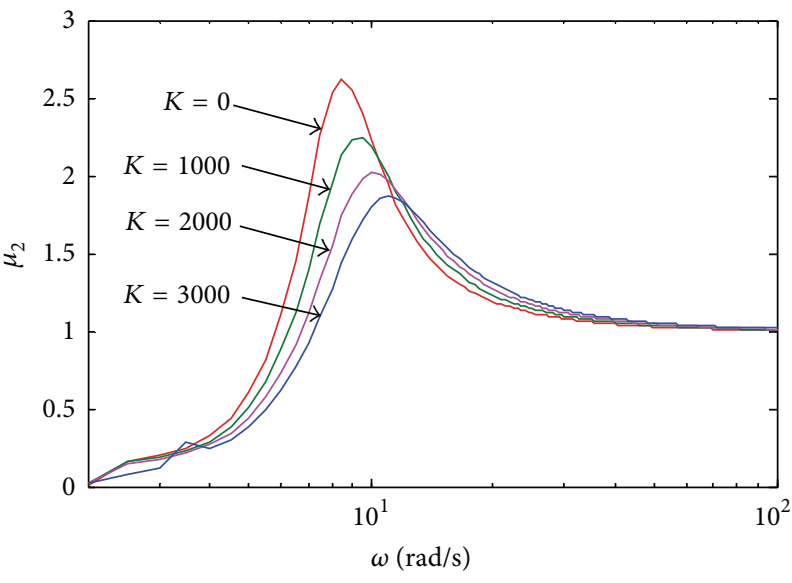

(a) Effect of $K$ on relative displacement transmissibility

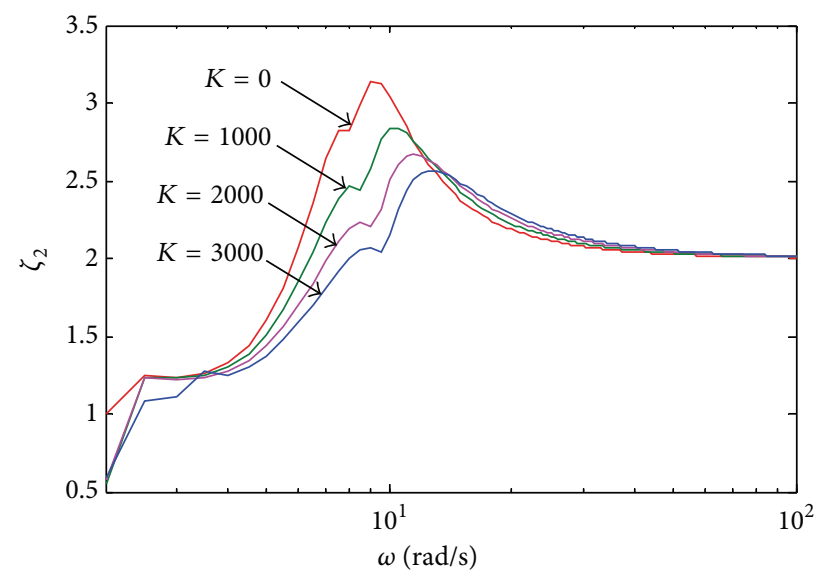

(b) Effect of $K$ on absolute displacement transmissibility

FIGURE 6: Effect of $K$ on the steady-state amplitudes under LRD control.

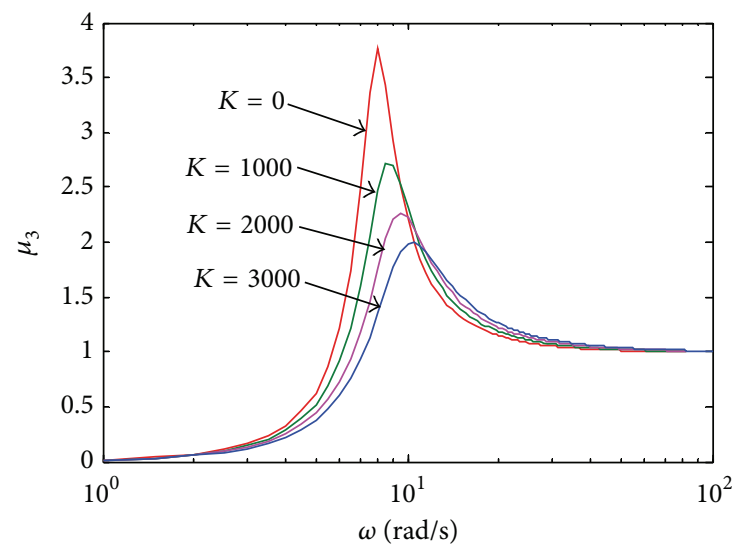

(a) Effect of $K$ on relative displacement transmissibility

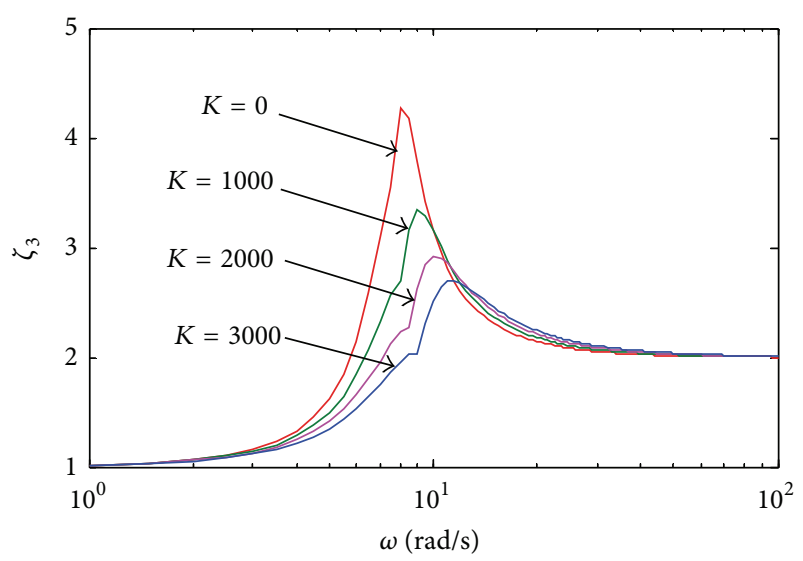

(b) Effect of $K$ on absolute displacement transmissibility

FIGURE 7: Effect of $K$ on the steady-state amplitudes under relative control. 


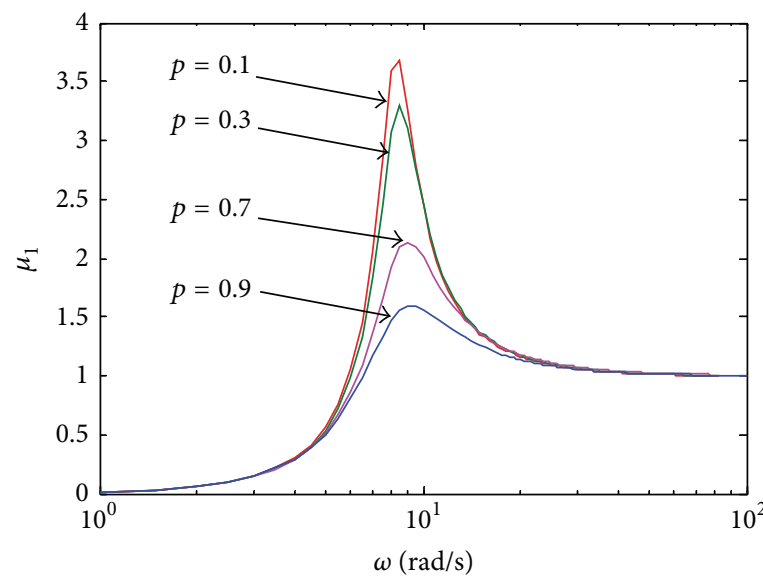

(a) Effect of $p$ on relative displacement transmissibility

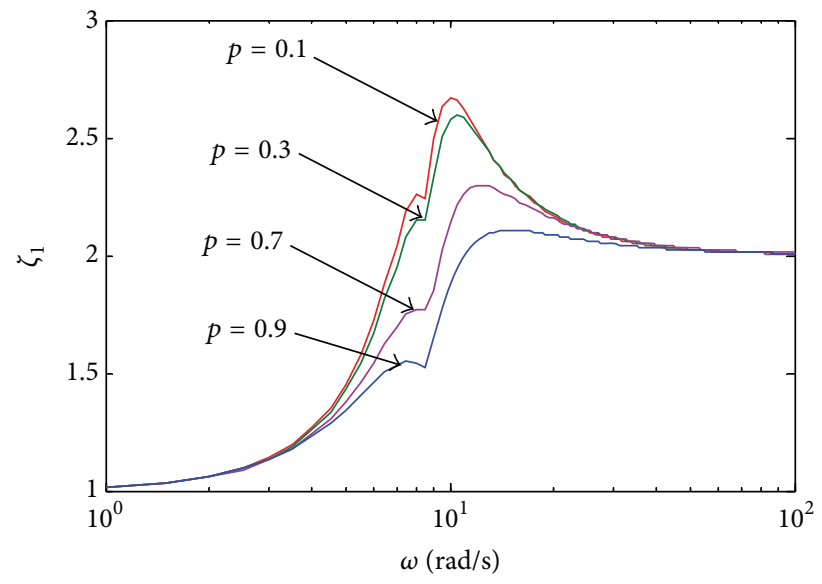

(b) Effect of $p$ on absolute displacement transmissibility

Figure 8: Effect of $p$ on the steady-state amplitudes under on-off control.

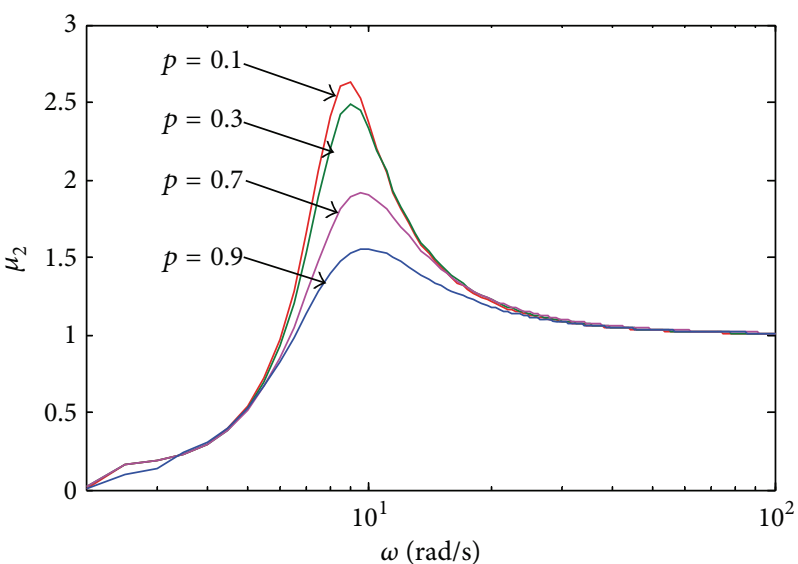

(a) Effect of $p$ on relative displacement transmissibility

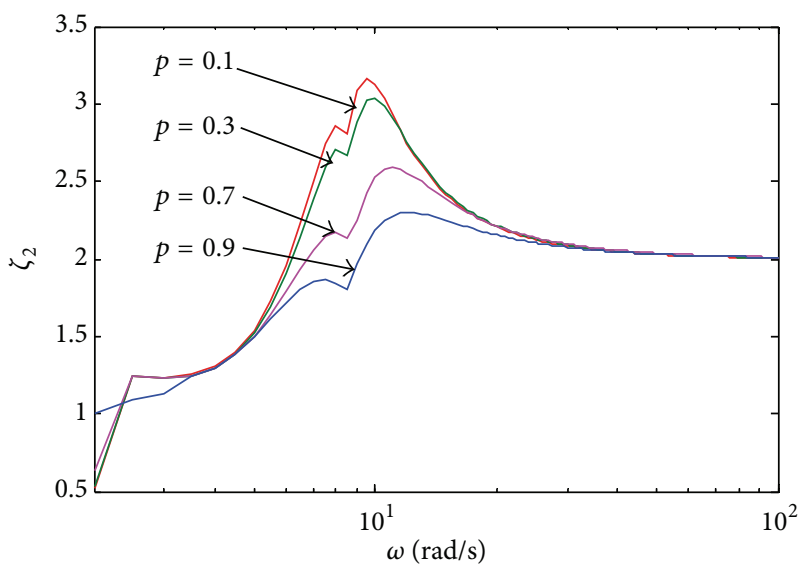

(b) Effect of $p$ on absolute displacement transmissibility

FIGURE 9: Effect of $p$ on the steady-state amplitudes under LRD control.

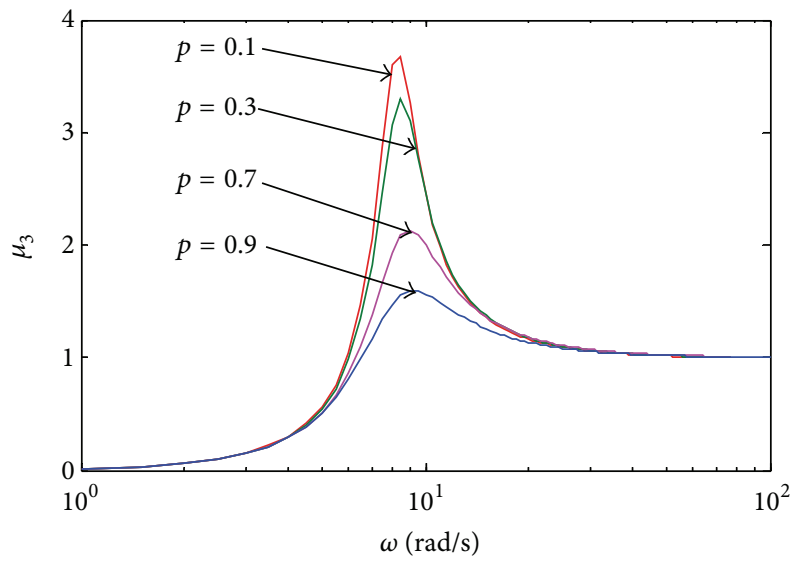

(a) Effect of $p$ on relative displacement transmissibility

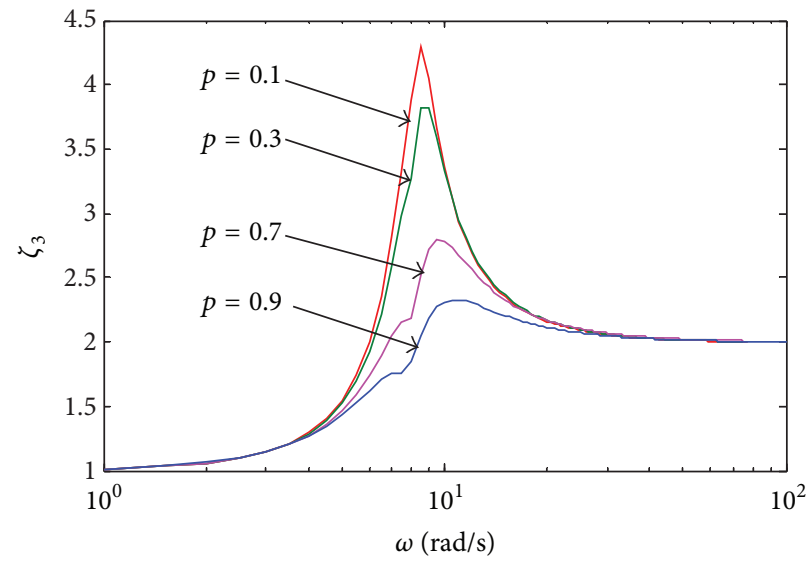

(b) Effect of $p$ on absolute displacement transmissibility

Figure 10: Effect of $K$ on the steady-state amplitudes under relative control. 


\section{Conflict of Interests}

The authors declare that there is no conflict of interests regarding the publication of this paper.

\section{Acknowledgments}

The authors are grateful to the support by National Natural Science Foundation of China (no. 11372198), the program for New Century Excellent Talents in university (NCET-11-0936), the cultivation plan for innovation team and leading talent in colleges and universities of Hebei Province (LJRC018), the program for advanced talent in the universities of Hebei Province (GCC2014053), and the program for advanced talent in Hebei Province (A201401001).

\section{References}

[1] J. P. Den Hartog, Mechanical Vibration, McGraw-Hill, New York, NY, USA, 1947.

[2] D. Karnopp, M. J. Crosby, and R. A. Harwood, "Vibration control using semi-active force generators," Journal of Manufacturing Science and Engineering, vol. 96, no. 2, pp. 619-626, 1974.

[3] J. K. Hedlick, "Railway vehicle active suspension," Vehicle System Dynamics, vol. 10, pp. 267-272, 1981.

[4] Y. J. Shen, S. P. Yang, C. Z. Pan, and H. J. Xing, "Semiactive control of hunting motion of locomotive based on magnetorheological damper," International Journal of Innovative Computing, Information and Control, vol. 2, no. 2, pp. 323-329, 2006.

[5] D. Karnopp, "Active and semi-active vibration isolation," ASME Journal of Mechanical Design, vol. 117, no. 6, pp. 177-185, 1995.

[6] S. M. Savaresi, V. C. Poussot, C. Spelta et al., Semi-Active Suspension Control Design for Vehicles, Elsevier, Amsterdam, The Netherlands, 2010.

[7] G. Emanuele, S. Tudor, W. S. Charles et al., Semi-Active Suspension Control-Improved Vehicle Ride and Road Friendliness, Springer, London, UK, 2008.

[8] C. Fabio, M. Georges, and M. Francesco, Technology of SemiActive Devices and Applications in Vibration Mitigation, John Wiley \& Sons, West Sussex, UK, 2008.

[9] Y. Shen, M. F. Golnaraghi, and G. R. Heppler, "Semi-active vibration control schemes for suspension systems using magnetorheological dampers," Journal of Vibration and Control, vol. 12, no. 1, pp. 3-24, 2006.

[10] N. Eslaminasab, O. Vahid A., and F. Golnaraghi, "Nonlinear analysis of switched semi-active controlled systems," Vehicle System Dynamics, vol. 49, no. 1-2, pp. 291-309, 2011.

[11] Y.-J. Shen, L. Wang, S.-P. Yang, and G.-S. Gao, "Nonlinear dynamical analysis and parameters optimization of four semiactive on-off dynamic vibration absorbers," Journal of Vibration and Control, vol. 19, no. 1, pp. 143-160, 2013.

[12] Y. J. Shen and M. Ahmadian, "Nonlinear dynamical analysis on four semi-active dynamic vibration absorbers with time delay," Shock and Vibration, vol. 20, no. 4, pp. 649-663, 2013.

[13] Y.-J. Shen, S.-P. Yang, H.-J. Xing, and C.-Z. Pan, "Analytical research on a single degree-of-freedom semi-active oscillator with time delay," Journal of Vibration and Control, vol. 19, no. 12, pp. 1895-1905, 2013.
[14] R. L. Bagley and P. J. Torvik, "Fractional calculus-a different approach to the analysis of viscoelastically damped structures," AIAA Journal, vol. 21, no. 5, pp. 741-748, 1983.

[15] R. L. Bagley, "Power law and fractional calculus model of viscoelasticity," AIAA Journal, vol. 27, no. 10, pp. 1412-1417, 1989.

[16] R. L. Bagley and P. J. Torvik, "A theoretical basis for the application of fractional calculus to viscoelasticity," Journal of Rheology, vol. 27, no. 3, pp. 201-210, 1983.

[17] Y. A. Rossikhin and M. V. Shitikova, "Application of fractional derivatives to the analysis of damped vibrations of viscoelastic single mass systems," Acta Mechanica, vol. 120, no. 1-4, pp. 109$125,1997$.

[18] M. S. Tavazoei, M. Haeri, M. Attari, S. Bolouki, and M. Siami, "More details on analysis of fractional-order Van der Pol oscillator," Journal of Vibration and Control, vol. 15, no. 6, pp. 803-819, 2009.

[19] C. M. A. Pinto and J. A. T. Tenreiro Machado, "Complex order van der Pol oscillator," Nonlinear Dynamics, vol. 65, no. 3, pp. 247-254, 2011.

[20] Y. J. Shen, S. P. Yang, H. J. Xing, and G. Gao, "Primary resonance of Duffing oscillator with fractional-order derivative," Communications in Nonlinear Science and Numerical Simulation, vol. 17, no. 7, pp. 3092-3100, 2012.

[21] Y. J. Shen, S. P. Yang, H. J. Xing, and H. Ma, "Primary resonance of Duffing oscillator with two kinds of fractional-order derivatives," International Journal of Non-Linear Mechanics, vol. 47, no. 9, pp. 975-983, 2012.

[22] Y.-J. Shen, P. Wei, and S.-P. Yang, "Primary resonance of fractional-order van der Pol oscillator," Nonlinear Dynamics, vol. 77, no. 4, pp. 1629-1642, 2014.

[23] Y. J. Shen, S. P. Yang, and C. Y. Sui, "Analysis on limit cycle of fractional-order van der Pol oscillator," Chaos, Solitons \& Fractals, vol. 67, pp. 94-102, 2014.

[24] P. Wahi and A. Chatterjee, "Averaging oscillations with small fractional damping and delayed terms," Nonlinear Dynamics, vol. 38, no. 1-4, pp. 3-22, 2004.

[25] L. C. Chen and W. Q. Zhu, "Stochastic jump and bifurcation of Duffing oscillator with fractional derivative damping under combined harmonic and white noise excitations," International Journal of Non-Linear Mechanics, vol. 46, no. 10, pp. 1324-1329, 2011.

[26] X. Y. Wang and Y. J. He, "Projective synchronization of fractional order chaotic system based on linear separation," Physics Letters, Section A: General, Atomic and Solid State Physics, vol. 372, no. 4, pp. 435-441, 2008.

[27] Z. L. Huang and X. L. Jin, "Response and stability of a SDOF strongly nonlinear stochastic system with light damping modeled by a fractional derivative," Journal of Sound and Vibration, vol. 319, no. 3-5, pp. 1121-1135, 2009.

[28] Y. Xu, Y. G. Li, D. Liu, W. Jia, and H. Huang, "Responses of Duffing oscillator with fractional damping and random phase," Nonlinear Dynamics, vol. 74, no. 3, pp. 745-753, 2013.

[29] I. Podlubny, Fractional Differential Equations, Academic Press, London, UK, 1998.

[30] A. A. Kilbas, H. M. Srivastava, and J. J. Trujillo, Theory and Applications of Fractional Differential Equations, Elsevier, Amsterdam, The Netherlands, 2006.

[31] S. Das, Functional Fractional Calculus for System Identification and Controls, Springer, Berlin, Germany, 2008.

[32] R. Caponetto, G. Dongola, L. Fortuna, and I. Petras, Fractional Order Systems: Modeling and Control Applications, World Scientific, River Edge, NJ, USA, 2010. 
[33] C. A. Monje, Y. Q. Chen, B. M. Vinagre, D. Y. Xue, and V. Feliu, Fractional-Order Systems and Controls: Fundamentals and Applications, Springer, London, UK, 2010.

[34] I. Petras, Fractional-Order Nonlinear Systems: Modeling, Anal$y$ sis and Simulation, Higher Education Press, Beijing, China, 2011.

[35] M. Ahmadian, "On the isolation properties of semiactive dampers," Journal of Vibration and Control, vol. 5, no. 2, pp. 217-232, 1999. 


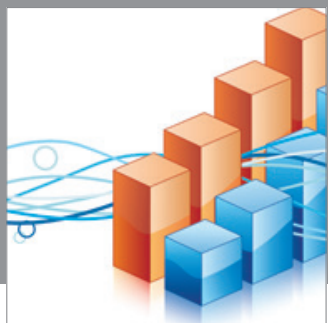

Advances in

Operations Research

mansans

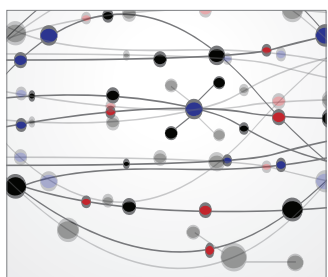

The Scientific World Journal
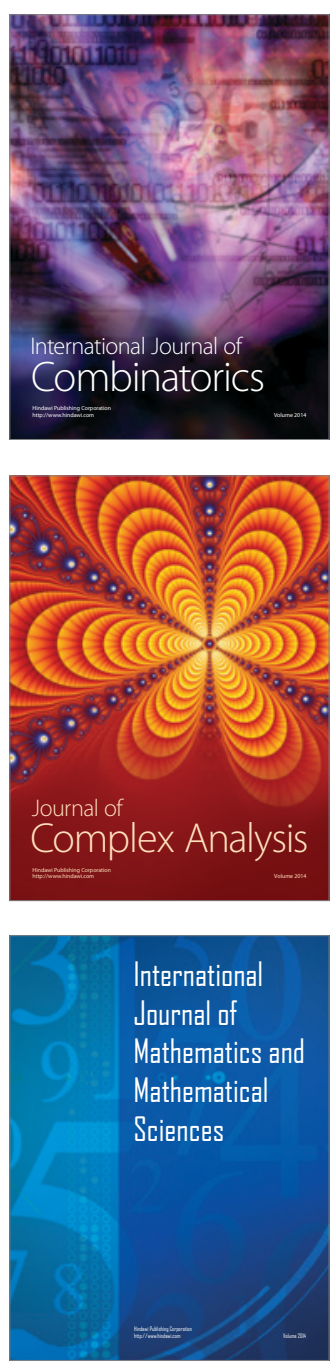
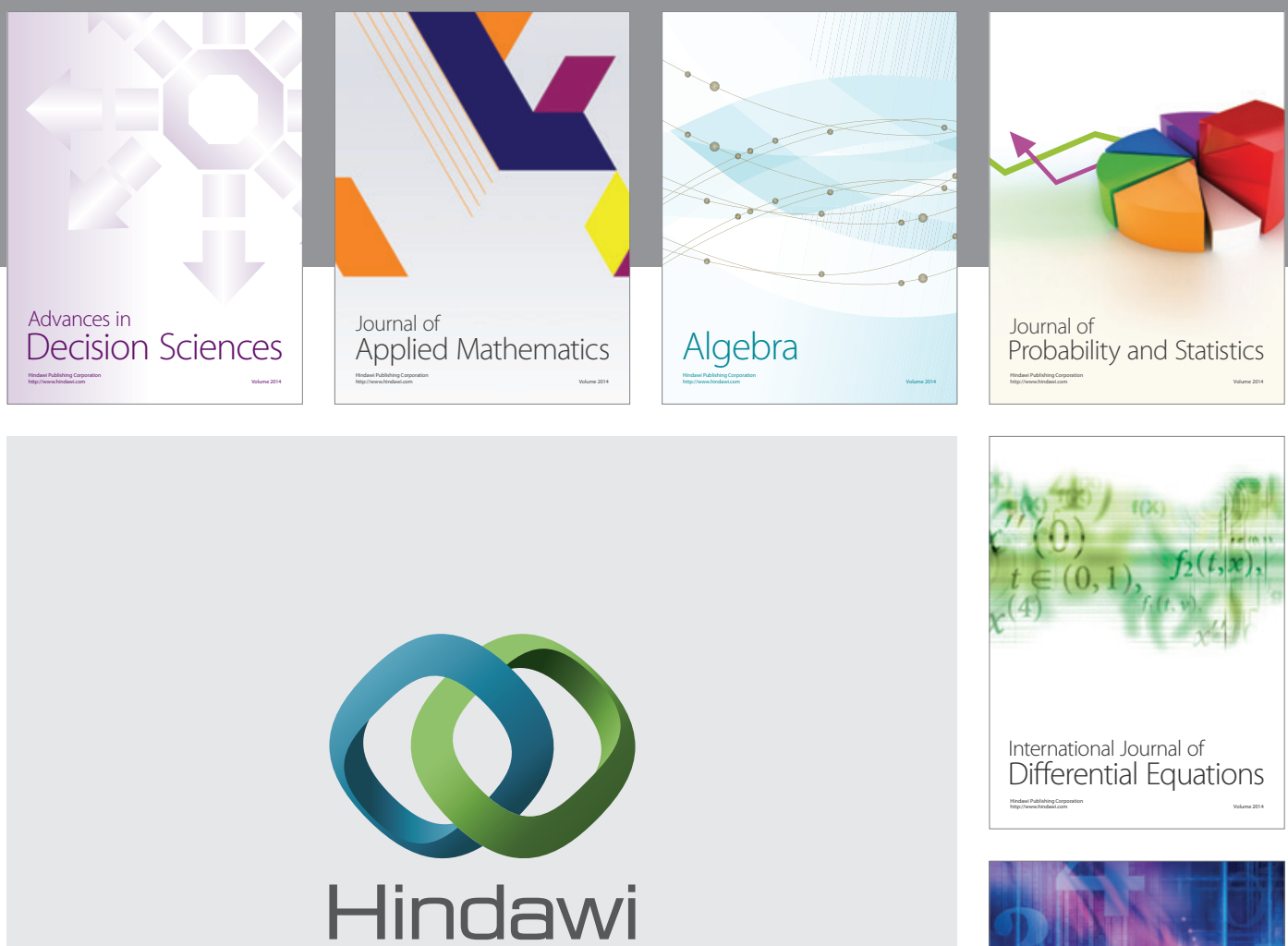

Submit your manuscripts at http://www.hindawi.com
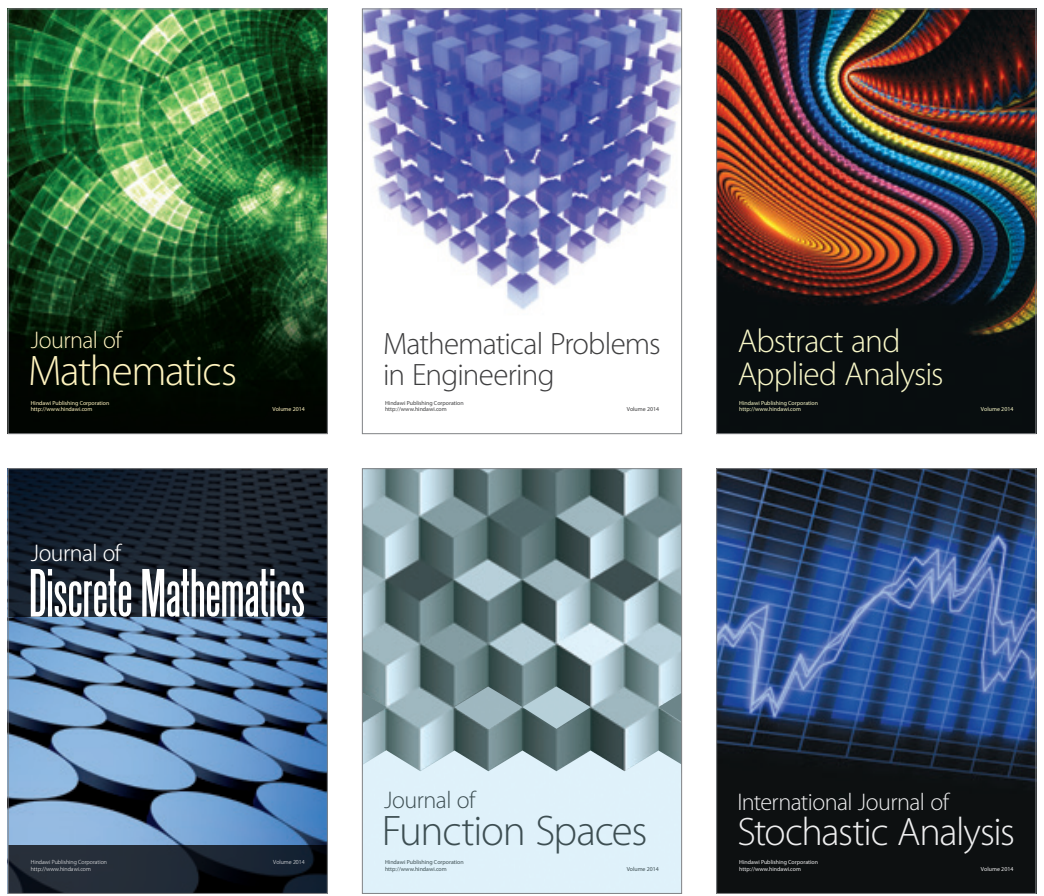

Journal of

Function Spaces

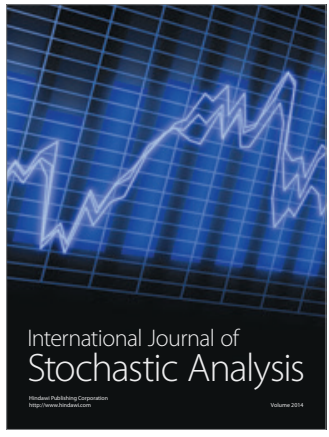

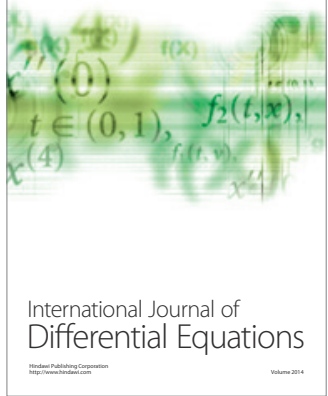
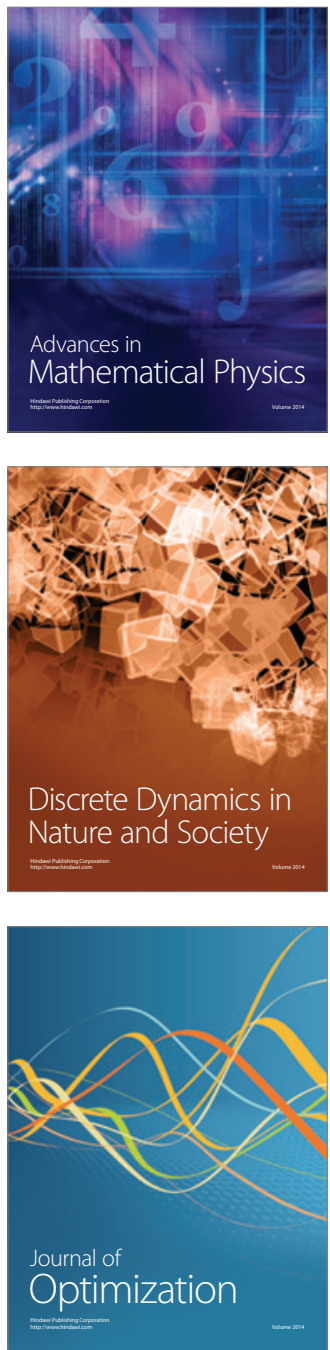